\title{
Hormone replacement therapy and patterns of osteoarthritis: baseline data from the Ulm Osteoarthritis Study
}

\author{
Andrea Erb, Hermann Brenner, Klaus-Peter Günther, Til Stürmer
}

\begin{abstract}
Objectives-It has been suggested that hormone replacement therapy (HRT) may protect against osteoarthritis (OA). The aim of this paper was to assess the association between HRT and radiographically defined patterns of OA.

Methods-475 consecutive women aged 50 years or older (mean age 66.1) who underwent hip or knee joint replacement because of advanced OA in four hospitals in south west Germany were enrolled in a cross sectional study. Participants underwent a standardised interview including detailed history of medication use and a physical examination. Furthermore, radiographs of the joint being replaced and of the contralateral joint as well as of both hands were obtained. Patients were categorised as having bilateral or unilateral OA according to the presence or absence of radiographic $O A$ in the contralateral joint. If radiographic OA of different hand and finger joint groups was present, participants were categorised as having generalised OA (GOA). Logistic regression was used to estimate odds ratios and their $95 \%$ confidence intervals for the association between HRT and bilateral or GOA while adjusting for potential confounders.
\end{abstract}

Results-Fifty five women $(11.6 \%)$ were using HRT. The median duration of use was 5.4 years. The prevalence of bilateral and GOA was similar among users of ORT $(86.3 \%$ and $27.5 \%$, respectively) and among non-users of HRT $(88.7 \%$ and $35.7 \%$, respectively). After adjustment for potential confounding factors, the odds ratios (95\% confidence intervals) of bilateral OA and GOA among HRT users compared with non-users was $1.21(0.48,3.03)$ and $1.21(0.53,2.74)$, respectively.

Conclusion-Despite limited generalisability because of the selective study sample, these data do not support the hypothesis that HRT acts as a systemic protective factor against $\mathrm{OA}$.

(Ann Rheum Dis 2000;59:105-109)

Osteoarthritis (OA) is the most common joint disorder and a major cause of disability in elderly people. Treatment options of OA are limited and concentrate on the relief of symptoms or joint replacement.

The use of hormone replacement therapy (HRT) after menopause has increased in recent years because of its beneficial effects on menopausal symptoms, bone mineral density and cardiovascular disease.

Sex hormones are likely to play a part in the development of OA in women. Before age 50, incidence of OA is much lower among women than among men. By contrast, the increase in incidence above age 50 is much steeper among women than among men. ${ }^{12}$ This suggests that the female oestrogen loss after menopause might be a risk factor for OA development. As HRT reduces various sequelae of oestrogen loss after menopause, it may protect against OA. On the other hand, HRT prevents bone loss after menopause, and a higher bone density was found to be prevalent in women with OA. ${ }^{3}$ According to the latter theory, HRT may be an indirect risk factor for OA.

Recent cross sectional studies have found an inverse association between ORT use and the prevalence of hip $\mathrm{OA}^{4}$ and knee OA. ${ }^{5}$ Hannan et al found a modest, but non-significant inverse association between oestrogen use and prevalence of knee $\mathrm{OA}$ in the Framingham study. ${ }^{6}$ A recent eight year follow up of the Framingham cohort as well as a four year follow up of the Chingford study indicated that current use of HRT had a moderate, but statistically non-significant protective effect against incident knee OA. ${ }^{78}$ In contrast, a nested casecontrol study could not confirm an association between current HRT use and the incidence of hip, knee and hand OA. ${ }^{9}$ However, a high OA incidence was found in new oestrogen users and the authors concluded that the utilisation of medical services by new HRT users increases the likelihood of an OA diagnosis. Concerning hand OA, no association with HRT use was observed in two cross sectional studies. $^{5} 10$

The aim of this study was to assess the relation between HRT and radiographically defined patterns of OA in a large sample of postmenopausal women participating in the Ulm Osteoarthritis Study.

\section{Methods}

STUDY DESIGN AND POPULATION

The main reason for data regarding the epidemiology of OA being sparse compared with other diseases with major public health impact is the difficulty of performing invasive diagnostics such as radiology in population samples (cross sectional or cohort studies) or healthy people (case-control studies). This difficulty can be circumvented to some degree if different OA patterns are studied among patients with
Accepted for publication 5 November 1999 
joint replacement because of OA. These patients are in close surveillance and radiographs of additional joints (contralateral hip or knee, hands) can more easily be obtained. Although this design does not permit comparison of persons with and without OA, comparisons of subgroups of people with unilateral or bilateral $\mathrm{OA}$ and localised or generalised $\mathrm{OA}$ (GOA) are possible and might provide clues as to the potential role of systemic risk factors in the disease process.

Following these considerations, the Ulm Osteoarthritis Study enrolled consecutive patients who were hospitalised for hip or knee joint replacement because of advanced OA. Details of the study design have been reported elsewhere. ${ }^{11}$ Briefly, four orthopaedic centres in south west Germany participated in the study. Between January 1995 and December 1996, 1037 patients under the age of 76 were eligible for recruitment. Among these, $212(20 \%)$ were operated on before they could be examined and $16(1.5 \%)$ refused to participate. After written informed consent, 809 patients were recruited in the study, of whom 504 were women. We were unable to assess menopausal status in 147 women with hysterectomy, because these women usually gave their age at surgery as menopausal age. As we felt potential bias by exclusion of these women to be more important than potential bias by excluding a few women with early menopause and including a few women with unknown menopausal status, we included all 475 women aged 50 years (mean age at menopause for women without hysterectomy) or more with available information on medication use in our current analysis.

DATA COLLECTION

Patients were interviewed and examined by trained physicians on the day before the joint replacement according to a standardised protocol. Information about age, height and weight, history of diabetes and gout, history of surgeries, current or past cigarette smoking, age at menopause and number of full term pregnancies was obtained.

$H R T$

The interview also included a detailed history of regular medication use during the past three months, including hormone preparations. Drug use and brand names were verified, if possible, by inspection of medication packages brought to the hospital. To estimate the duration of use, the starting date of the medication was asked for.

$\mathrm{Up}$ to three active ingredients for up to 15 preparations were classified according to the ATC classification (WHO Collaborating Centre for Drug Statistics Methodology, Oslo, 1993).

HRT use was defined as regular use of any drug containing postmenopausal oestrogens in the past three months. HRT was furthermore classified according to whether progesteron was administered together with oestrogen or not. Analyses were confined to oral, transdermal and intramuscular applications. Topical oestrogens or vaginal suppositories were not considered because their systemic effects are expected to be negligible.

Radiographic assessment of $O A$

Standard radiographs were taken for the joint being replaced, the contralateral joint and both hands. Patients with hip OA underwent a radiographic examination consisting of a supine anteroposterior pelvic view, while patients with knee OA had anteroposterior weight bearing and lateral non-weight bearing radiographs of both knees. All hip and knee radiographs were read by one trained senior orthopaedic surgeon (KPG) without further information about the patient. Hip or knee OA was classified according to the Kellgren and Lawrence score from grade 0 (no OA) to 4 (advanced OA) using a standard reference atlas. ${ }^{12}$ Grade 0 and 1 were considered as normal, whereas grade 2 or higher was defined to represent manifest $\mathrm{OA}$. The reliability of radiographic grading of OA according to the Kellgren and Lawrence score on the hip and knee joints was assessed in 100 radiographs and was found to be high (inter-rater agreement between multiple orthopaedic surgeons: intraclass correlation coefficient $(\mathrm{ICC})=0.88$ for hip $\mathrm{OA}$ and 0.81 for knee $\mathrm{OA}$; intra-rater agreement for the rater of this study: $\mathrm{ICC}=0.88$ for hip $\mathrm{OA}$ and 0.93 for knee OA) ${ }^{13}{ }^{14}$ All patients had OA of the replaced joint. If OA was also present in the joint contralateral to the one being replaced, the patient was classified having a bilateral OA.

Hand radiographs were read by another trained orthopaedic surgeon without further information about the patient. All visible finger joints (proximal interphalangeal, PIP, and distal interphalangeal, DIP) and the carpometacarpal joints (CMC) were evaluated regarding osteophytes, joint space narrowing and subchondral sclerosis. A definite joint space narrowing or possible joint space narrowing in combination with definite osteophytes and/or subchondral sclerosis were considered as OA. This score puts more weight on joint space narrowing than on osteophytes, because osteophytes are often absent in finger joints despite joint space narrowing and sclerosis, and because joint space narrowing has been shown to have better reproducibility than osteophytes. ${ }^{15}$ If OA was found in two or more finger joints and in one or both CMC joints in addition to OA of the replaced joint, the patient was considered to have GOA. ${ }^{11}{ }^{16}$ The reliability of this classification was assessed in 50 pairs of hand radiographs and was found to be sufficiently good (inter-rater agreement: $\kappa=0.73$; intra-rater agreement for the rater of this study: $\kappa=0.54$ ).

STATISTICAL ANALYSES

We first carried out descriptive analyses on basic sociodemographic variables, HRT use, and the prevalence of bilateral OA and GOA in the study population. We assessed the difference in the prevalence of possible confounding factors between HRT users and non-users and its large sample $95 \%$ confidence interval. We then compared the prevalence of bilateral OA and GOA between users and non-users of HRT using multivariable logistic regression to adjust the association between HRT use and 
Table 1 Description of the study population

\begin{tabular}{ll}
\hline & $n(\%)$ \\
\hline Number & 475 \\
Age & \\
Mean & 66.1 \\
Median & 67 \\
Interquartile range & $62-71$ \\
HRT users & $55(11.6 \%)$ \\
Oestrogen mono-therapy & $29(52.7 \%)$ \\
Oestrogen-gestagen combinations & $26(47.3 \%)$ \\
Hip OA & $194(40.8 \%)$ \\
Knee OA & $281(59.2 \%)$ \\
Radiographs of the contralateral joint & $432(90.9 \%)$ \\
Bilateral OA & $382(88.2 \%)$ \\
Hand radiographs & $376(79.2 \%)$ \\
Generalised OA $\dagger$ & $131(34.7 \%)$ \\
\hline
\end{tabular}

^Defined as radiological OA (Kellgren and Lawrence score $\geqslant 2$ ) of replaced joint and contralateral joint. †Defined as radiological $\mathrm{OA}$ on $\geqslant 2$ finger joints and $\geqslant 1$ carpometacarpal joint in addition to uni- or bilateral OA of hip or knee.

bilateral or GOA for potential confounding factors. The following potential confounders that are known or suspected risk factors of OA were considered: age (continuous), body mass index (BMI: weight divided by squared height, continuous), diabetes mellitus (history or use of oral antidiabetics or insulin), gout (history or use of uricostatic or uricosuric medication), thiazide intake, hysterectomy status and smoking. All analyses were performed with the SAS statistical software package (Version 6.12, SAS Institute Inc, Cary, NC).

\section{Results}

The mean age of the study participants was 66.1 years. Overall, 55 of 475 women were using HRT (table 1). Of these, 29 were taking oestrogen only preparations and 26 used oestrogengestagen combinations. The median duration of use was 5.4 years with an interquartile range from 2.3 to 8 years. One hundred and ninety four women had prevalent hip OA, and 281 women had prevalent knee OA. Radiographs of the contralateral joint were available for $91 \%$ of the women ( $9 \%$ refused additional radiographs of the contralateral joint), and a bilateral hip or knee joint OA was observed in the majority of these patients $(88.2 \%)$. For $79 \%$ of the women, radiographs of the hands were available (missing values attributable to patients refusing additional hand radiographs) and $34.7 \%$ of these had a GOA.

Table 2 shows the distribution of potential confounding variables according to the use of HRT. Looking at the median age, HRT users were seven years younger than non-users of HRT. Women with oestrogen intake were less likely to have diabetes and more likely to be current smokers and having had a hysterectomy. There were no major differences between the groups regarding body mass index, age at menopause, gout and thiazide use.

In table 3 we present the number of participants and the prevalence of bilateral and GOA according to oestrogen intake, followed by the crude and adjusted odds ratios (OR) and their 95\% confidence intervals (CI) for the association between HRT use and the different OA patterns. Odds ratios above one correspond to a higher prevalence of bilateral or GOA in HRT users compared with non-users.
Women using HRT had a slightly lower prevalence of bilateral OA $(86.3 \%)$ than women not using HRT $(88.7 \%)$. This was mainly attributable to confounding by age, however. After adjustment for age and the other covariates, there was a weak, statistically non-significant positive association between oestrogen use and bilateral $\mathrm{OA}(\mathrm{OR}=1.21$; 95\% CI: 0.48, 3.03).

Users of HRT had a somewhat lower prevalence of GOA $(27.5 \%)$ compared with nonusers $(35.7 \%)$ resulting in a crude odds ratio of 0.68 (95\% CI:0.33, 1.42). Again, the crude odds ratio was strongly confounded by age. After adjustment for confounding, there was a weak, statistically non-significant positive association between HRT and GOA (OR=1.21; 95\% CI: 0.53, 2.74).

Conducting the analysis according to the duration of current oestrogen use, we found no major differences in the prevalences of generalised or bilateral OA between women who used HRT five years or more, less than five years, and non-users (table 4), but the small numbers in the subgroups have to be taken into account. Looking at women with use of oestrogen mono-preparations and oestrogen-gestagen combinations separately, we found no significant differences between either of the two subgroups in comparison with non-users concerning both bilateral and GOA (data not shown).

\section{Discussion}

In contrast with previous studies reporting a protective effect of HRT on knee or hip OA, we observed no association between HRT use and either bilateral knee or hip OA or GOA in the Ulm Osteoarthritis Study.

Two recent cross sectional studies found an inverse association between HRT and prevalent hip ${ }^{4}$ or knee ${ }^{5}$ OA. In the Chingford Study ${ }^{5}$ 45-64 year old women were examined, and current users of HRT (for more than one year) showed a lower prevalence of knee OA, which was defined by osteophytes, than non-users $(\mathrm{OR}=0.31 ; 95 \% \mathrm{CI}: 0.11,0.93)$. In the Study of Osteoporotic Fractures ${ }^{4}$ participants 65 years or older were included. An inverse association of HRT use with hip OA, which was defined on a radiographic summary score, ${ }^{17}$ was observed (OR=0.63; 95\% CI: $0.49,0.86)$. In 600 women aged 63 or older from the Framingham OA Study, women who ever used HRT for more than two years showed a one third lower prevalence of radiographically defined knee OA, but the difference from nonusers was statistically non-significant. ${ }^{6}$ This inverse association was stronger looking at women with bilateral OA only (OR $=0.52 ; 95 \%$ CI: $0.25,1.09)$. In contrast with the cross sectional studies, the exposure in the Framingham Study was assessed before the outcome making a bias of reversed causality less likely.

Two recent population based prospective studies also suggested a moderate protective effect of HRT regarding the incidence of knee OA, but the confidence intervals around these estimates were wide and included the null value. In an eight year follow up of the Framingham cohort, a possible reduction of 
Table 2 Distribution of possible confounding variables according to HRT

\begin{tabular}{|c|c|c|c|}
\hline & \multicolumn{2}{|l|}{$H R T^{\star}$} & \multirow[b]{2}{*}{ Difference $(95 \%$ CI) } \\
\hline & yes & no & \\
\hline Number & 55 & 420 & \\
\hline \multicolumn{4}{|l|}{ Age } \\
\hline Median & 61 & 68 & \\
\hline Interquartile range & $57-66$ & $64-72$ & \\
\hline \multicolumn{4}{|l|}{$\mathrm{BMI}+$} \\
\hline Median & 27.5 & 28.2 & \\
\hline Interquartile range & $24.7-30.2$ & $25.7-31.6$ & \\
\hline \multicolumn{4}{|l|}{ Age at menopause $\ddagger$} \\
\hline Median & 51 & 50 & \\
\hline Interquartile range & $50-54$ & $48-53$ & \\
\hline \multicolumn{4}{|l|}{ Prevalence of $(\%)$} \\
\hline Current smoking & 10.9 & 4.8 & $6.1(-2.3$ to 14.6$)$ \\
\hline Former smoking & 18.2 & 18.8 & $-0.6(-11.5$ to 10.2$)$ \\
\hline Diabetes mellitus $₫$ & 1.8 & 10.5 & $-8.7(-13.3$ to -4.1$)$ \\
\hline Gout & 14.5 & 10.3 & $4.2(-5.5$ to 14.0$)$ \\
\hline Thiazide intake & 14.4 & 19.8 & $-5.4(-15.3$ to 4.8$)$ \\
\hline Hysterectomy & 41.8 & 27.6 & $14.2(0.5$ to 27.9$)$ \\
\hline
\end{tabular}

${ }^{\star}$ Use of hormone replacement therapy. †Body mass index $\left(\mathrm{kg} / \mathrm{m}^{2}\right)$. $\ddagger$ Based on 347 women with available information on age at menopause. \History of diabetes or use of oral antidiabetics or insulin. $\uparrow$ History of gout or use of uricosurica/uricostatica.

risk of incident radiographically defined knee $\mathrm{OA}$ was found in current HRT users $(\mathrm{OR}=0.4$; $95 \%$ CI: $0.1,3.0) .{ }^{7} \mathrm{~A}$ four year follow up of the Chingford population was compatible with a protective effect of current HRT use on the development of knee osteophytes ( $\mathrm{OR}=0.4$ : $95 \%$ CI $0.1,1.4$ ), but also with an increase in risk regarding the development of joint space narrowing $(\mathrm{OR}=1.9 ; 95 \% \mathrm{CI}: 0.9,4.1){ }^{8}$

HRT use was not associated with hand OA in the Chingford study. ${ }^{5}$ In this study, DIP and CMC OA were defined according to the Kellgren and Lawrence score and considered separately. Samanta et al observed no association between HRT and prevalent GOA, defined by Herberden's nodes and polyarticular interphalangeal OA, in a British case-control study. ${ }^{18}$

A recent case-control study did not find an association between current HRT use and incident cases of hip, knee and hand $\mathrm{OA},{ }^{9}$ which was defined as radiographic OA according to the Kellgren and Lawrence score plus symptoms. However, a high OA incidence was found in new oestrogen users, and the authors concluded that the utilisation of medical services by new HRT users increases the likelihood of an OA diagnosis. A further casecontrol study found a slightly positive associa- tion between women who ever used ORT for less than five years and advanced symptomatic hip OA, which might reflect a possibly higher prescription rate of oestrogens to women with present hip pain. ${ }^{19}$

In the previous studies, women with OA were compared with women without OA. In contrast, participants of our study were selected according to a manifest OA of one hip or knee joint. This selection makes different access to medical care unlikely. The selection of our study participants who all had advanced $\mathrm{OA}$ in one hip or knee joint is likely to be independent of the different OA patterns, the laterality or generalisation. On the other hand, results of our study with participants preselected by the presence of advanced OA in at least one joint may not be generalisable to early forms or incident $\mathrm{OA}$.

OA patterns in our study were classified radiographically after documentation of a very good (hip and knee joint) or sufficient (GOA) intra-rater and inter-rater reliability. Classification of the participants into patients with unilateral or bilateral OA was based on the radiograph of the joint opposite to the one being replaced. The classification into generalised or localised $\mathrm{OA}$ is equivalent to the definition of the Chingford study for knee OA, where GOA was defined by the presence of OA in both finger joints (DIP and PIP) and carpometacarpal joint in addition to knee OA. ${ }^{16}$ This definition was extended to hip OA in our study, because the hip joints are-like the knee joints - big, weight bearing joints. ${ }^{1120}$ Together with hip or knee OA, these women had radiological OA of at least three different joint groups. The overall prevalence of GOA might not be directly comparable to other studies using different radiographic gradings of hand OA, but this should not affect the validity of the internal comparisons (odds ratios) presented.

Detailed history of current medication use including hormone preparations was asked for by a trained physician and confirmed by medication packages brought to the hospital. An investigation of the Postmenopausal Estrogen/ Progestin Interventions Trial showed a 95\% agreement concerning the reporting of an ever

Table 3 Patterns of $O A$ according to HRT

\begin{tabular}{|c|c|c|c|c|c|c|}
\hline \multirow[b]{3}{*}{$H R T$} & \multicolumn{3}{|l|}{ Bilateral $O A$} & \multicolumn{3}{|l|}{ Generalised $O A$} \\
\hline & \multirow[b]{2}{*}{ prevalence } & \multicolumn{2}{|l|}{ Odds ratio $(95 \% \mathrm{CI})$} & \multirow[b]{2}{*}{ prevalence } & \multicolumn{2}{|l|}{ Odds ratio $(95 \%$ CI) } \\
\hline & & crude & adjusted $^{\star}$ & & crude & adjusted $^{\star}$ \\
\hline no & $88.7 \%(338 / 381)$ & 1 (reference) & 1 (reference) & $35.7 \%(120 / 336)$ & 1 (reference) & 1 (reference) \\
\hline yes & $86.3 \%(44 / 51)$ & $0.80(0.34$ to 1.89$)$ & 1.21 (0.48 to 3.03$)$ & $27.5 \%(11 / 40)$ & $0.68(0.33$ to 1.42$)$ & $1.21(0.53$ to 2.74$)$ \\
\hline
\end{tabular}

${ }^{\star}$ Adjusted for age, body mass index, diabetes mellitus, gout, thiazide intake, hysterectomy and smoking.

Table 4 Patterns of $O A$ according to duration of current HRT use

\begin{tabular}{|c|c|c|c|c|c|c|}
\hline \multirow{3}{*}{$\begin{array}{l}\text { Duration of } \\
\text { HRT use }\end{array}$} & \multicolumn{3}{|l|}{ Bilateral $O A$} & \multicolumn{3}{|l|}{ Generalised $O A$} \\
\hline & \multirow[b]{2}{*}{ prevalence } & \multicolumn{2}{|l|}{ Odds ratio $(95 \%$ CI) } & \multirow[b]{2}{*}{ prevalence } & \multicolumn{2}{|l|}{ Odds ratio $(95 \% \mathrm{CI})$} \\
\hline & & crude & adjusted $^{*}$ & & crude & adjusted $^{\star}$ \\
\hline 0 years & $88.7 \%(338 / 381)$ & 1 (reference) & 1 (reference) & $35.7 \%(120 / 336)$ & 1 (reference) & 1 (reference) \\
\hline$<5$ years & $83.3(20 / 24)$ & $0.64(0.21$ to 1.95$)$ & $0.91(0.28$ to 3.00$)$ & $26.3 \%(5 / 19)$ & $0.64(0.23$ to 1.83$)$ & $1.13(0.36$ to 3.60$)$ \\
\hline $5+$ years & $88.9(24 / 27)$ & $1.02(0.29$ to 3.52$)$ & $1.60(0.44$ to 5.83$)$ & $28.6 \%(6 / 21)$ & $0.72(0.27$ to 1.90$)$ & $1.28(0.45$ to 3.66$)$ \\
\hline
\end{tabular}

${ }^{\star}$ Adjusted for age, body mass index, diabetes mellitus, gout, thiazide intake, hysterectomy and smoking. 
use of HRT. ${ }^{21}$ As HRT use is socially well accepted it is unlikely that oestrogens are deliberately not reported in our study.

We controlled for a variety of possible confounders using multivariable logistic regression. We adjusted for age, body mass index, diabetes mellitus, gout, age of menopause, thiazide intake, hysterectomy status and smoking. Unlike other studies we did not control for bone mineral density because this may be on the causal pathway between ORT and OAthat is, oestrogen users are less affected by loss of bone mineral density after menopause than non-users and bone mineral density might be associated with the risk of OA. ${ }^{322}$ In our study, age was the main confounder of the association between HRT and different OA patterns. This results from the fact that HRT use decreases with age, while the prevalence of OA, especially GOA, is increasing.

A limitation of our study is that information was obtained for current regular medication during the past three months only. Hence, there was no information about past HRT use and the group of non-users may have included some women who have used HRT in the past. Their number should be very small, however, given that HRT use was very rare in Germany in the past. For example, in a national survey conducted eight years before our study, only $4.5 \%$ of the women between $45-80$ years (mean age 58.1 years) were currently taking HRT. ${ }^{23}$ Therefore, possible bias attributable to missing information about past HRT use is probably small. One further possibility may be that some women stopped HRT intake before surgery because of anxiety about an increased risk of thrombosis, but it is unlikely that they stopped oestrogen medication three month before surgery, which was the time period hormone use was asked for. In current users of HRT, we had no information about the type and duration of use of different preparations in the past in case of a previous change in preparation, nor about compliance. Therefore, the results regarding duration of use have to be interpreted keeping this limitation in mind. Concerning the duration of use, a mean of a five year use might be too short to see an effect of HRT on a slowly progressive disease like OA. Furthermore, we could not investigate the time sequence between HRT and OA patterns because of the cross sectional study design. Therefore, the possibility of an OA manifestation before the beginning of HRT use cannot be ruled out. Besides, the possibility that HRT may be used by women to relief symptoms of painful joints cannot be excluded.

In summary, we did not observe an independent association between HRT and either generalised or bilateral OA in postmenopausal women with advanced OA of the hip or knee joint. Despite its limitations, our study does not support the hypothesis that HRT could act as a systemic protective factor against OA.

We would like to thank the participating institutions, Departments of Orthopaedic Surgery, (head: Dr W Puhl) and Trauma Surgery (Dr L Kinzl) of the University of Ulm, Hessing Ortho- paedic Hospital in Augsburg (Dr Th Naumann), and Baumann Orthopaedic Hospital in Stuttgart (Dr C T Trepte) for their effort in recruiting the patients and Ms B Mack for data processing. We would also like to thank Dr P Dieppe for his ideas and support in developing the radiographic hand score. Funding: the study was supported by a grant from the German
Federal Ministry for Research and Technology (BMFT 01 FF940/6)

1 Oliveria SA, Felson DT, Reed JI, Cirillo PA, Walker AM. Incidence of symptomatic hand, hip and knee osteoarthritis among patients in a health maintenance organisation. tis among patients in a health maint

2 Sun Y, Stürmer T, Günther KP, Brenner H. Incidence and prevalence of osteoarthritis of the hip and knee in the general population [in German]. Z Orthop 1997;135:184-92.

3 Hart DJ, Mootoosamy I, Doyle DV, Spector TD. The relationship between osteoarthritis and osteoporosis in the general population: The Chingford Study. Ann Rheum Dis 1994;53:158-62.

4 Nevitt MC, Cummings SR, Lane NE, Hochberg MC, Scott JC, Pressman MA, et al. Association of estrogen replacement therapy with the risk of osteoarthritis of the hip in elderly white women. Arch Intern Med 1996;156:2073-80.

5 Spector TD, Nandra D, Hart DJ, Doyle DV. Is hormone replacement therapy protective for hand and knee osteoarthritis in women? The Chingford study. Ann Rheum Dis 1997;56:432-4.

6 Hannan MT, Felson DT, Anderson JJ, Naimark A, Kannel WB. Estrogen use and radiographic osteoarthritis of the knee in women: The Framingham Osteoarthritis Study. Arthritis Rheum 1990;33:525-32.

7 Zhang Y, McAlindon TE, Hannan MT, Chaisson CE, Klein R, Wilson PWF, et al. Estrogen replacement therapy and worsening of radiographic knee osteoarthritis: the Framworsening of radiographic knee osteoarthritis: the
ingham Study. Arthritis Rheum 1998;41:1867-73.

8 Hart DJ, Doyle DV, Spector TD. Incidence and risk factors for radiographic knee osteoarthritis in middle-aged women: the Chingford Study. Arthritis Rheum 1999;42: $17-24$.

9 Oliveria SA, Felson DT, Klein RA, Reed JI, Walker AM. Estrogen replacement therapy and the development of osteoarthritis. Epidemiology 1996;7:415-19.

10 Sowers MF, Hochberg M, Crabbe JP, Muhich A, Crutchfield M, Updike S. Association of bone mineral density and sex $M$, Updike S. Association of bone mineral density and sex
hormone levels with osteoarthritis of the hand and knee in premenopausal women. Am J Epidemiol 1996;143:38-47. premenopausal women. Am J Epidemiol 1996;143:38-47.
Günther KP, Stürmer T, Sauerland S, Zeissig I, Sun Y, Kessler S, et al. Prevalence of generalized osteoarthritis in patients with advanced hip and knee osteoarthritis: The Ulm Osteoarthritis Study. Ann Rheum Dis 1998;57:717-23.

12 Empire Rheumatism Council. The epidemiology of chronic rheumatism. In: Atlas of standard radiographs of arthritis. Vol 2, Oxford: Blackwell Scientific Publications, 1963.

13 Günther KP, Scharf HP, Puhl W, Willauschus W, Kalke Y, Glückert K, et al. Reliability of radiographic assessment in Glückert K, et al. Reliability of radiographic assessment in
osteoarthritis of the knee joint [in German]. Z Orthop 1997;135:197-202.

14 Günther KP, Scharf HP, Puhl W, Willauschus W, Sauerland $\mathrm{S}$, Glückert K, et al. Reproducibility of radiographic evaluation in osteoarthritis of the hip [in German]. Z Orthop 1997;135:3-8.

15 Kallman DA, Wigley FM, Scott WW, Hochberg MC, Tobin JD. New radiographic scales for osteoarthritis of the hand. Arthritis Rheum 1989;32:1584-91.

16 Hart DJ, Spector TD, Egger P, Coggon D, Cooper C. Defining osteoarthritis of the hand for epidemiological studies: the Chingford study. Ann Rheum Dis 1994;53: 220-3.

17 Croft P, Cooper C, Wickham C, Coggon D. Defining osteoarthritis of the hip for epidemiologic studies. Am J Epidemiol 1990;132:514-22.

18 Samanta A, Jones A, Regan M, Wilson S, Doherty M. Is osteoarthritis in women affected by hormonal changes or smoking? J Rheumatol 1993;32:366-70.

19 Dennison EM, Arden NK, Kellingray S, Croft P, Coggon D, Cooper C. Hormone replacement therapy, other reproductive variables and symptomatic hip osteoarthritis in elderly white women: a case-control study. Br J Rheumatol 1998;37:1198-1202.

20 Stürmer T, Sun Y, Sauerland S, Zeißig I, Günther KP, Puhl $\mathrm{W}$, et al. Serum cholesterol and osteoarthritis. Results from the baseline examination of the Ulm Osteoarthritis Study. J Rheumatol 1998;25:1827-32.

21 Greendale GA, James MK, Espeland MA, Barrett-Connor E. Can we measure prior estrogen/progestin use? The Postmenopausal Estrogen/Progestin Interventions Trial. The PEPI investigators. Am J Epidemiol 1997;146:763-70.

22 Hannan MT, Anderson JJ, Zhang Y, Levy D, Felson DT. Bone mineral density and knee osteoarthritis in elderly
men and women. The Framingham Study. Arthritis men and women. The

23 Weyermann M, Brenner H. Factors affecting bone demineralization and blood lead levels of postmenopausal women a population-based study from Germany. Environ Res 1998;76:19-25. 